\title{
Front Matter: Volume 7598
}

, "Front Matter: Volume 7598," Proc. SPIE 7598, Optical Components and Materials VII, 759801 (1 April 2010); doi: 10.1117/12.862538

SPIE. Event: SPIE OPTO, 2010, San Francisco, California, United States 


\section{PROCEEDINGS OF SPIE}

\section{Optical Components and Materials VII}

Shibin Jiang

Michel J. F. Digonnet

John W. Glesener

J. Christopher Dries

Editors

26-28 January 2010

San Francisco, California, United States

Sponsored and Published by

SPIE

Volume 7598 
The papers included in this volume were part of the technical conference cited on the cover and title page. Papers were selected and subject to review by the editors and conference program committee. Some conference presentations may not be available for publication. The papers published in these proceedings reflect the work and thoughts of the authors and are published herein as submitted. The publisher is not responsible for the validity of the information or for any outcomes resulting from reliance thereon.

Please use the following format to cite material from this book:

Author(s), "Title of Paper," in Optical Components and Materials VII, edited by Shibin Jiang, Michel J. F. Digonnet, John W. Glesener, J. Christopher Dries, Proceedings of SPIE Vol. 7598 (SPIE, Bellingham, WA, 2010) Article CID Number.

ISSN 0277-786X

ISBN 9780819479945

Published by

SPIE

P.O. Box 10, Bellingham, Washington 98227-0010 USA

Telephone +1 3606763290 (Pacific Time) · Fax +1 3606471445

SPIE.org

Copyright (C) 2010, Society of Photo-Optical Instrumentation Engineers

Copying of material in this book for internal or personal use, or for the internal or personal use of specific clients, beyond the fair use provisions granted by the U.S. Copyright Law is authorized by SPIE subject to payment of copying fees. The Transactional Reporting Service base fee for this volume is $\$ 18.00$ per article (or portion thereof), which should be paid directly to the Copyright Clearance Center (CCC), 222 Rosewood Drive, Danvers, MA 01923. Payment may also be made electronically through CCC Online at copyright.com. Other copying for republication, resale, advertising or promotion, or any form of systematic or multiple reproduction of any material in this book is prohibited except with permission in writing from the publisher. The CCC fee code is 0277-786X/10/\$18.00.

Printed in the United States of America.

Publication of record for individual papers is online in the SPIE Digital Library.

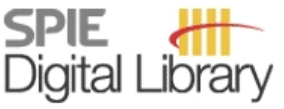

SPIEDigitalLibrary.org

Paper Numbering: Proceedings of SPIE follow an e-First publication model, with papers published first online and then in print and on CD-ROM. Papers are published as they are submitted and meet publication criteria. A unique, consistent, permanent citation identifier (CID) number is assigned to each article at the time of the first publication. Utilization of CIDs allows articles to be fully citable as soon they are published online, and connects the same identifier to all online, print, and electronic versions of the publication. SPIE uses a six-digit CID article numbering system in which:

- The first four digits correspond to the SPIE volume number.

- The last two digits indicate publication order within the volume using a Base 36 numbering system employing both numerals and letters. These two-number sets start with 00, 01, 02, 03, 04 , 05, 06, 07, 08, 09, OA, OB ... 0Z, followed by 10-1Z, 20-2Z, etc.

The CID number appears on each page of the manuscript. The complete citation is used on the first page, and an abbreviated version on subsequent pages. Numbers in the index correspond to the last two digits of the six-digit CID number. 


\section{Contents}

xi Conference Committee

\section{SESSION 1 GAIN MATERIALS I}

759802 Similar behaviors of sulfide and selenide-based chalcogenide glasses to form glass ceramics (Invited Paper) [7598-01]

L. Calvez, Lab. des Verres et Céramiques, CNRS, Univ. de Rennes 1 (France); C. Lin, Wuhan Univ. of Technology (China); M. Rozé, Lab. des Verres et Céramiques, CNRS, Univ. de Rennes 1 (France); Y. Ledemi, Lab. dos Materials Fôtonicos, Instituto de Quimica (Brazil);

E. Guillevic, B. Bureau, Lab. des Verres et Céramiques, CNRS, Univ. de Rennes 1 (France); M. Allix, CEMHTI, CNRS, Univ. d'Orléans (France); X. Zhang, Lab. des Verres et Céramiques, CNRS, Univ. de Rennes 1 (France)

759803 The influence of photonic mode density on the luminescence of erbium-doped optical materials (Invited Paper) [7598-02]

F. Song, Nankai Univ. (China) and Key Lab. of Weak Light Nonlinear Photonics (China); Q. Wang, Nankai Univ. (China), Key Lab. of Weak Light Nonlinear Photonics (China), and City Univ. of Hong Kong (Hong Kong, China); C. Ming, J. Tian, J. XU, Nankai Univ. (China) and Key Lab. of Weak Light Nonlinear Photonics (China); S. Lin, E. Y. B. Pun, City Univ. of Hong Kong (Hong Kong, China)

759804 Two-photon pumped random lasing in a dye-doped silica gel powder [7598-03]

S. García-Revilla, Univ. del País Vasco (Spain); Í. Solá, Univ. de Salamanca (Spain); R. Balda, Univ. del País Vasco (Spain); L. Roso, Univ. de Salamanca (Spain); D. Levy, M. Zayat, Instituto de Ciencia de Materiales de Madrid (Spain); J. Fernández, Univ. del País Vasco (Spain)

759805 Spectral and luminescent properties of Bi-doped bulk glasses and factors acting on them [7598-04]

B. I. Denker, B. I. Galagan, I. L. Shulman, S. E. Sverchkov, A.M. Prokhorov General Physics Institute (Russian Federation); E. M. Dianov, Fiber Optics Research Ctr. (Russian Federation)

759806 Generation of wide color gamut visible light in NIR-excited thulium-holmium-ytterbium codoped tantalum oxide nanopowders [7598-48]

A. S. Gouveia-Neto, L. A. Bueno, E. B. Costa, E. A. Silva, Jr., Univ. Federal Rural de Pernambuco (Brazil); J. L. Ferrari, K. O. Lima, R. R. Gonçalves, Univ. de São Paulo (Brazil)

\section{SESSION 2 GAIN MATERIALS II}

759807 Application of ceramic phosphors for near infrared biomedical imaging technologies (Invited Paper) [7598-05]

K. Soga, K. Tokuzen, K. Tsuji, T. Yamano, N. Venkatachalam, H. Hyodo, H. Kishimoto, Tokyo Univ. of Science (Japan) 
759808 White LED phosphors: the next step (Invited Paper) [7598-06]

H. Yamamoto, Tokyo Univ. of Technology (Japan)

759809 Construction of photoconductivity measurement system as functions of excitation wavelength and temperature: application to Eu2+-activated phosphors [7598-07] T. Nakanishi, S. Tanabe, Kyoto Univ. (Japan)

7598 OA The efficiencies of energy transfer from $\mathrm{Cr}$ to $\mathrm{Nd}$ ions in silicate glasses [7598-08] S. Mizuno, H. Ito, K. Hasegawa, Toyota Central R\&D Labs., Inc. (Japan); H. Nasu, M. Hughes, T. Suzuki, Y. Ohishi, Toyota Technological Institute (Japan)

$7598 \mathrm{OB}$ The influence of $\mathrm{Yb}^{2+}$ ions on optical properties and power stability of ytterbium-doped laser fibers [7598-09]

J. Kirchhof, S. Unger, A. Schwuchow, S. Jetschke, V. Reichel, M. Leich, A. Scheffel, Institute of Photonic Technology, Jena (Germany)

\section{SESSION 3 SILICON TECHNOLOGY}

7598 OC Silicon photonic parametric optical processing for ultra-high bandwidth on-chip signal grooming (Invited Paper) [7598-10]

A. Biberman, N. Ophir, K. Bergman, Columbia Univ. (United States)

7598 OD Optical properties of atomic layer deposited materials and their application in silicon waveguides [7598-11]

T. Alasaarela, Helsinki Univ. of Technology (Finland); J. Hiltunen, VTT Technical Research Ctr. of Finland (Finland); A. Khanna, A. Säynätjoki, A. Tervonen, S. Honkanen, Helsinki Univ. of Technology (Finland)

7598 OE Silicon based 2-dimensional slot waveguides [7598-12]

A. Khanna, T. Alasaarela, A. Säynätjoki, A. Tervonen, S. Honkanen, Helsinki Univ. of Technology (Finland)

7598 OF Optical microresonator for application to an opto-electronic oscillator [7598-13]

Y.-M. WU, L. Vivien, E. Cassan, Institut d'Électronique Fondamentale, CNRS, Univ. Paris Sud 11 (France); V. H. N. Luong, L. D. Nguyen, B. Journet, SATIE, CNRS, École Normale Supérieure de Cachan (France)

\section{SESSION 4 OPTICAL FIBERS AND DEVICES I}

7598 OG Recent developments in bend-insensitive and ultra-bend-insensitive fibers [7598-14] D. Boivin, L.-A. de Montmorillon, L. Provost, N. Montaigne, Draka Communications (France); F. Gooijer, E. Aldea, J. Jensma, Draka Communications (Netherlands); P. Sillard, Draka Communications (France)

$7598 \mathrm{OH}$ Innovative fiber coating systems based on organic modified ceramics [7598-15] K. Schuster, J. Kobelke, Institute for Photonic Technology, Jena (Germany); K. Rose, Fraunhofer-Institut für Silicatforschung (Germany); M. Helbig, SurA Chemicals GmbH (Germany); M. Zoheidi, A. Heinze, FiberTech GmbH (Germany) 
7598 ol Novel shape memory alloy optical fibre connection method [7598-16]

G. Trovillard, P. Zivojinovic, R. Cerutti, X. P. Godmaire, E. Weynant, Phasoptx Inc. (Canada)

7598 0J Tunable birefringent phase shift induced in fiber Bragg gratings by a shape memory alloy phase modulator [7598-17]

A. Fraser, PhasOptx (Canada) and Univ. Laval (Canada); M. Bernier, Univ. Laval (Canada);

E. Weynant, PhasOptx Inc. (Canada); R. Vallée, Univ. Laval (Canada)

7598 OK Novel fiber bottle microresonator add-drop filters [7598-18]

G. Senthil Murugan, J. S. Wilkinson, M. N. Zervas, Univ. of Southampton (United Kingdom)

SESSION 5 OPTICAL FIBERS AND DEVICES II

$7598 \mathrm{OL} \quad$ Ultra-wideband integrated 2x2 optical router using novel MMI design [7598-19]

M. A. Swillam, M. H. Bakr, X. Li, McMaster Univ. (Canada)

$759800 \quad$ Fabrication of low losses chalcogenide photonic crystal fibers by molding process [7598-22]

Q. Coulombier, Sciences Chimiques de Rennes, CNRS, Univ. de Rennes 1 (France);

L. Brilland, PERFOS (France); P. Houizot, Sciences Chimiques de Rennes, CNRS, Univ. de

Rennes 1 (France); T. N. Nguyen, T. Chartier, Ecole Nationale Supérieure des Sciences

Appliquées et de Technologie (France); G. Renversez, Institut Fresnel, CNRS, Univ. St Jérôme (France); A. Monteville, PERFOS (France); J. Fatome, F. Smektala, Lab. Interdisciplinaire Carnot de Bourgogne, CNRS, Univ. de Bourgogne (France); T. Pain, Sciences Chimiques de Rennes, CNRS, Univ. de Rennes 1 (France); H. Orain, J.-C. Sangleboeuf, Lab. de Recherche en Mécanique Appliquée, CNRS, Univ. de Rennes 1 (France); J. Trolès, Sciences Chimiques de Rennes, CNRS, Univ. de Rennes 1 (France)

SESSION 6 PHOTONIC DEVICES I

7598 OP Fabrication and characterization of colloidal crystals infiltrated with metallic nanoparticles [7598-23]

A. Chiappini, CNR-IFN, CSMFO Lab. (Italy); S. Guddala, CNR-IFN, CSMFO Lab. (Italy), Univ. of Hyderabad (India), and Univ. degli Studi di Trento (Italy); C. Armellini, CNR-IFN, CSMFO Lab. (Italy) and FBK (Italy); S. Berneschi, I. Cacciari, MDF Lab., Istituto di Fisica Applicata Nello Carrara, CNR (Italy); C. Duverger-Arfuso, Lab. LdOF, CNRS, Univ. du Maine (France); M. Ferrari, CNR-IFN, CSMFO Lab. (Italy); G. C. Righini, MDF Lab., Istituto di Fisica Applicata Nello Carrara, CNR (Italy)

$75980 Q \quad$ Planar long-period gratings for photonic applications [7598-24]

J. Jiang, C. L. Callender, C. J. Ledderhof, Communications Research Ctr. Canada (Canada); J. Ding, National Research Council Canada (Canada)

7598 OR Time evolution of an electro-optic modulator by detection of its nonlinear behavior [7598-25]

D. T. Bui, L. D. Nguyen, SATIE, CNRS, École Normale Supérieure de Cachan (France); I. Ledoux-Rak, LPQM, CNRS, École Normale Supérieure de Cachan (France); B. Journet, SATIE, CNRS, École Normale Supérieure de Cachan (France)

7598 OS Compact Raman spectrometer system for low frequency spectroscopy [7598-26]

C. Moser, F. Havermeyer, Ondax, Inc. (United States) 
7598 OT A new class of polarization filters for laser applications [7598-27]

L. Wang, C. Hodgson, T. Erdogan, Semrock, Inc. (United States)

SESSION 7 PHOTONIC DEVICES II

7598 OV Serial pixel analog-to-digital converter (ADC) [7598-30]

E. D. Larson, National Security Technologies, LLC (United States)

\section{SESSION 8 DETECTOR TECHNOLOGIES I}

7598 OX Simulation based design for back-side illuminated ultrahigh-speed CCDs [7598-32]

T. Arai, T. Hayashida, K. Kitamura, J. Yonai, H. Maruyama, NHK Science and Technology Research Labs. (Japan); N. Ohtsuka, C. Vo Le, T. G. Etoh, Kinki Univ. (Japan); H. van Kuijk, DALSA Corp. (Netherlands)

7598 OY Modified uni-traveling-carrier photodiode-based V-band optoelectronic mixers with high up-converted power and high responsivity [7598-33]

H. Pan, Z. Li, J. C. Campbell, Univ. of Virginia (United States)

$75980 Z$ Dark count in single photon avalanche Si detectors [7598-34]

R. Pagano, Univ. degli Studi di Catania (Italy) and Istituto per la Microelettronica e Microsistemi, CNR (Italy); S. Libertino, Istituto per la Microelettronica e Microsistemi, CNR (Italy); G. Valvo, G. Condorelli, B. Carbone, A. Piana, M. Mazzillo, D. N. Sanfilippo, G. G. Fallica, STMicroelectronics (Italy); G. Falci, Univ. degli Studi di Catania (Italy) and MATIS, CNR (Italy); S. Lombardo, Istituto per la Microelettronica e Microsistemi, CNR (Italy)

\section{SESSION 9 DETECTOR TECHNOLOGIES II}

759811 DC and AC performance of leaky-mode metal-semiconductor-metal polysilicon photodetectors [7598-36]

R. Pownall, Colorado State Univ. (United States); G. Yuan, Avago Technologies Ltd. (United States); C. Thangaraj, J. Kindt, T. W. Chen, Colorado State Univ. (United States); P. Nikkel, Avago Technologies Ltd. (United States); K. L. Lear, Colorado State Univ. (United States)

759812 Ultra-high sensitivity photodetector arrays with integrated amplification and passivation nano-layers (Invited Paper) [7598-37]

J. Yao, I. A. Mokina, F. Liu, S. Wang, J. Zhou, B\&W Tek, Inc. (United States); M. Lange, Princeton Univ. (United States); W. Yang, P. Gardner, Western Carolina Univ. (United States); L. Peltz, R. Frampton, J. H. Hunt, The Boeing Co. (United States)

759813 Back-thinned CMOS sensor optimization [7598-75]

P. Jerram, D. Burt, N. Guyatt, e2v technologies plc (United Kingdom); V. Hibon, J. Vaillant, Y. Henrion, e2v semiconductors SAS (France)

759814 Two micron pore size MCP-based image intensifiers [7598-38]

J. Glesener, J. Estrera, L-3 Electro-Optical Systems (United States) 
759815 Progress in crystalline semiconductor core optical fibers (Invited Paper) [7598-40] J. Ballato, T. Hawkins, P. Foy, C. McMillen, R. Stolen, Clemson Univ. (United States); R. Rice, Northrop Grumman Space Technology (United States)

759817 Pulse amplification in semiconductor optical amplifiers with ultrafast gain-recovery times [7598-42]

P. P. Baveja, A. M. Kaplan, Institute of Optics, Univ. of Rochester (United States);

D. N. Maywar, Rochester Institute of Technology (United States); G. P. Agrawal, Institute of Optics, Univ. of Rochester (United States)

759818 High-temperature stability of lasing wavelength in GaAsSb/GaAs double quantum wells lasers [7598-43]

H.-C. Yu, C.-T. Wan, Y.-K. SU, R. W. Chuang, W.-C. Chen, C.-Y. Huang, W.-H. Lin, M. H. Pilkuhn, National Cheng Kung Univ. (Taiwan)

$759819 \quad 1.54 \mu \mathrm{m}$ emitter and optical amplifier based on Er doped InGaN/GaN [7598-44]

R. Dahal, J. Y. Lin, H. X. Jiang, Texas Tech Univ. (United States); J. M. Zavada, North Carolina State Univ. (United States)

\section{SESSION 11 LASER AND AMPLIFIERS I}

7598 1A Near and medium infrared optical fiber lasers and emerging applications (Invited Paper) [7598-45]

F. Prudenzano, L. Mescia, L. Allegretti, M. De Sario, A. D'Orazio, A. Di Tommaso, T. Palmisano, V. Petruzzelli, Politecnico di Bari (Italy)

7598 1B Chromium-doped zinc selenide gain media: from synthesis to pulsed mid-infrared laser operation (Invited Paper) [7598-46]

A. Sennaroglu, Koç Univ. (Turkey); U. Demirbas, Koç Univ. (Turkey) and Massachusetts Institute of Technology (United States); H. Cankaya, N. Cizmeciyan, Koç Univ. (Turkey); A. Kurt, Teknofil, Inc. (Turkey); M. Somer, Koç Univ. (Turkey)

7598 1C Pulsed cladding-pumped large mode area fiber Raman amplifier [7598-47] J. Ji, C. A. Codemard, J. Sahu, J. Nilsson, Univ. of Southampton (United Kingdom)

\section{SESSION 12 LASER AND AMPLIFIERS II}

7598 1D Rogue waves in femtosecond supercontinuum generation [7598-49] M. Erkintalo, G. Genty, Tampere Univ. of Technology (Finland); J. M. Dudley, Univ. de Franche-Comté (France)

7598 IE Terrace-microsphere lasers: spherical cavity lasers for multiwavelength emission [7598-50] H. Uehara, T. Yano, S. Shibata, Tokyo Institute of Technology (Japan) 
7598 1H Direct surface relief formation in As-S(Se) layers [7598-53]

M. Trunov, Uzhgorod National Univ. (Ukraine); P. Lytvyn, V. Lashkaryov Institute of Semiconductor Physics (Ukraine); P. M. Nagy, Central Institute of Chemical Researches (Hungary); Cs. Cserhati, I. Charnovich, S. Kokenyesi, The Univ. of Debrecen (Hungary)

7598 1J Potentials of the acousto-optical spectral data processing on a basis of a novel algorithm of the collinear wave heterodyning in a large-aperture KRS-5 crystalline cell [7598-55] A. S. Shcherbakov, Instituto Nacional de Astrofísica, Óptica y Electrónica (Mexico); J. Maximov, Molecular Technology GmbH (Germany); D. Sanchez Lucero, Instituto Nacional de Astrofísica, Óptica y Electrónica (Mexico)

$75981 \mathrm{~K}$ Some peculiarities of designing the optical scheme of tellurium dioxide crystalline cell based acousto-optical spectrometer for the Mexican Large Millimeter Telescope [7598-56] A. S. Shcherbakov, D. Sanchez Lucero, A. Luna Castellanos, Instituto Nacional de Astrofísica, Óptica y Electrónica (Mexico); J. Maximov, Molecular Technology GmbH (Germany)

7598 IL Excitation wavelength dependence of quantum efficiencies of $\mathrm{Nd}$-doped glasses for solar pumped fiber lasers [7598-57]

T. Suzuki, H. Kawai, H. Nasu, M. Hughes, Toyota Technological Institute (Japan); S. Mizuno,

K. Hasegawa, H. Ito, Toyota Central Research and Development Labs., Inc. (Japan);

Y. Ohishi, Toyota Technological Institute (Japan)

$75981 \mathrm{M}$ Ultraflat supercontinuum generation in an $\mathrm{As}_{2} \mathrm{~S}_{3}$-based chalcogenide core microstructured fiber [7598-58]

X. Yan, C. Chaudhari, G. Qin, M. Liao, T. Suzuki, Y. Ohishi, Toyota Technological Institute (Japan)

7598 iN Optical parametric amplification in composite tellurite-fluorophosphate fiber [7598-59]

C. Chaudhari, T. Suzuki, Y. Ohishi, Toyota Technological Institute (Japan)

$7598 \mathrm{IP} \quad$ Electronic polarizability and optical parameters of $\mathrm{Er}^{3+} / \mathrm{Yb}^{3+} \mathrm{co}$-doped phosphate glasses [7598-61]

F. Song, C. Ming, W. Wang, L. Luo, Nankai Univ. (China)

7598 IR Polymer microlens array with tunable focal intensity by the polarization control of the incident light [7598-63]

S.-Y. Huang, T.-C. Tung, S.-W. Ko, C.-L. Ting, H.-C. Jau, M.-S. Li, H.-C. Lin, A. Y.-G. Fuh, National Cheng Kung Univ. (Taiwan)

7598 is Fabrication of one-dimensional SWS on bismuth borate glass by glass-imprinting method [7598-64]

N. Yamashita, Isuzu Glass Co., Ltd. (Japan) and National Institute of Advanced Industrial Science and Technology (Japan); T. Suetsugu, Isuzu Glass Co., Ltd. (Japan); N. Kitamura, K. Fukumi, National Institute of Advanced Industrial Science and Technology (Japan); J. Nishii, Hokkaido Univ. (Japan)

7598 1T A small and fast SCPEM-based ellipsometer [7598-65]

F. Bammer, Technische Univ. Wien (Austria); R. Petkovšek, J. Možina, J. Petelin, Univ. of Ljubljana (Slovenia) 
7598 IW Birefringence control of pigtails in fiber optic devices [7598-68]

D. Tentori, Ctr. de Investigación Científica y de Educación Superior de Ensenada (Mexico); C. Ayala-Diaz, Univ. Autónoma de Baja California (Mexico); F. Treviño Martinez, Univ. Autonoma de Nuevo Leon (Mexico)

7598 1X AIN antiresonant layer ARROW waveguides [7598-69]

M. V. Pelegrini, D. O. Carvalho, M. I. Alayo, I. Pereyra, Univ. de São Paulo (Brazil)

7598 IY Steep and flat bandpass filter using linearly chirped and apodized fiber Bragg grating [7598-70]

X. Wu, J. Jacquet, Supélec, Lab. Matériaux Optiques Photonique et Systèmes, CNRS (France); G. Duan, Alcatel-Thalès III-V Lab. (France)

759820 Spectroscopy of Yb:Im doped tellurite glasses for efficient infrared fiber laser [7598-72] H. Gebavi, Politecnico di Torino (Italy); M. Taher, Swansea Univ. (United Kingdom); J. Lousteau, D. Milanese, Politecnico di Torino (Italy); S. Taccheo, Swansea Univ. (United Kingdom); A. Schulzgen, College of Optical Sciences, The Univ. of Arizona (United States); M. Ferraris, Politecnico di Torino (Italy); N. Peyghambarian, College of Optical Sciences, The Univ. of Arizona (United States)

$759821 \quad$ NIR to visible upconversion in rare-earth lon-doped NaYF 4 crystals [7598-73] D. Patel, C. Vance, N. King, M. Jessup, L. Green, Oakwood Univ. (United States); S. Sarkisov, SSS Optical Technologies (United States)

759822 Detector development for x-ray imaging [7598-76]

M. A. Mentzer, U.S. Army Aberdeen Test Ctr. (United States); D. A. Herr, Jacobs Aberdeen Test Support Services (United States); K. J. Brewer, U.S. Army Aberdeen Test Ctr. (United States); N. Ojason, H. A. Tarpine, Jacobs Aberdeen Test Support Services (United States)

Author Index 
Downloaded From: https://www.spiedigitallibrary.org/conference-proceedings-of-spie on 26 Apr 2023

Terms of Use: https://www.spiedigitallibrary.org/terms-of-use 


\title{
Conference Committee
}

\author{
Symposium Chair \\ E. Fred Schubert, Rensselaer Polytechnic Institute (United States) \\ Symposium Cochairs \\ Liang-Chy Chien, Kent State University (United States) \\ James G. Grote, Air Force Research Laboratory (United States) \\ Conference Chairs
}

Shibin Jiang, AdValue Photonics, Inc. (United States)

Michel J. F. Digonnet, Stanford University (United States)

John W. Glesener, L-3 Electro-Optical Systems (United States)

J. Christopher Dries, DOLCE Technologies, LLC (United States)

Program Track Chair

James G. Grote, Air Force Research Laboratory (United States)

Program Committee

Jean-Luc Adam, Université de Rennes 1 (France)

Robert P. Dahlgren, University of California, Santa Cruz (United States) and Silicon Valley Photonics, Ltd (United States)

Leonid B. Glebov, CREOL, The College of Optics and Photonics, University of Central Florida (United States)

Min Gu, Swinburne University of Technology (Australia)

Seppo K. Honkanen, Helsinki Universitetet of Technology (Finland)

Jacques Lucas, Université de Rennes 1 (France)

John R. Marciante, University of Rochester (United States)

Yasutake Ohishi, Toyota Technological Institute (Japan)

Aydogan Ozcan, University of California, Los Angeles (United States)

Barrett G. Potter, Jr., The University of Arizona (United States)

Giancarlo C. Righini, Istituto di Fisica Applicata Nello Carrara (Italy)

Stan M. Smith, U.S. Army Space and Missile Defense Command (United States)

Feng Song, Nankai University (China)

Setsuhisa Tanabe, Kyoto University (Japan)

Ji Wang, Corning Inc. (United States)

John M. Zavada, U.S. Army Research Office (United States) 
Session Chairs

1 Gain Materials I

Joaquín Fernández, Universidad del País Vasco (Spain)

2 Gain Materials II

Aydogan Ozcan, University of California, Los Angeles (United States)

3 Silicon Technology

John W. Glesener, L-3 Electro-Optical Systems (United States)

4 Optical Fibers and Devices I

Jie Yao, B\&W Tek, Inc. (United States)

5 Optical Fibers and Devices II

Michel J. F. Digonnet, Stanford University (United States)

6 Photonic Devices I

Michel J. F. Digonnet, Stanford University (United States)

7 Photonic Devices II

Qing Wang, AdValue Photonics, Inc. (United States)

8 Detector Technologies I

John W. Glesener, L-3 Electro-Optical Systems (United States)

9 Detector Technologies II

John W. Glesener, L-3 Electro-Optical Systems (United States)

10 Semiconductor Lasers and Amplifiers

Xianghua Zhang, Université de Rennes 1 (France)

11 Laser and Amplifiers I

Michel J. F. Digonnet, Stanford University (United States)

12 Laser and Amplifiers II

Jean-Luc Adam, Université de Rennes 1 (France) 